\title{
EXTENSIONS AND LIFTINGS OF POSITIVE LINEAR MAPPINGS ON BANACH LATTICES
}

\author{
BY
}

\author{
HEINRICH P. LOTZ( $\left.{ }^{1}\right)$
}

\begin{abstract}
Let $F$ be a closed sublattice of a Banach lattice $G$. We show that any positive linear mapping from $F$ into $L^{1}(\mu)$ or $C(X)$ for a Stonian space $X$ has a positive norm preserving extension to $G$. A dual result for positive norm preserving liftings is also established. These results are applied to obtain extension and lifting theorems for order summable and majorizing linear mappings. We also obtain some partial results concerning positive extensions and liftings of compact linear mappings.
\end{abstract}

The main purpose of this paper is to establish some conditions under which positive linear mappings between Banach lattices have norm preserving positive linear extensions or liftings. These results are then applied to obtain extension and lifting theorems for order summable and majorizing linear mappings and to establish the inductive and projective character of the $|\sigma|$ tensor product topology introduced by Jacobs [6]. Finally, we obtain some partial results concerning the problem of finding positive, compact linear extensions and liftings of positive compact linear mappings between Banach lattices.

We begin our discussion with a summary of known results concerning the corresponding problems for continuous linear mappings between Banach spaces. Suppose that $E, F$ and $G$ are Banach spaces, that $\phi$ is an isometry of $F$ into $G$ and that $T$ is a continuous linear mapping of $F$ into $E$. When does $T$ have a norm preserving linear extension $\hat{T}: G \rightarrow E$, that is, when does there exist a continuous linear mapping $\hat{T}$ of $G$ into $E$ such that $\|\hat{T}\|=\|T\|$ and such that the following diagram commutes?

If $E$ is the scalar field, the Hahn-Banach theorem asserts that such an extension $\hat{T}$ of $T$ always exists. Results of Nachbin [10], Kelley [9] and Goodner

Received by the editors June 26, 1972 and, in revised form, May 1, 1974. AMS (MOS) subject classifications (1970). Primary 47A20, 47B55, 47B05, 46M10, 46M05; Secondary 46A40, 46E99, 18G05.

Key words and phrases. Extensions and liftings of positive linear mappings, injective Banach lattices, order summable mappings, majorizing mappings, tensor products of Banach lattices.

(1) This research was supported by the National Science Foundation under Grant GP 28577. 


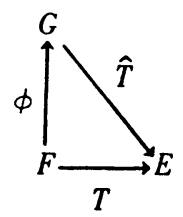

Figure 1

[4] show that, for a fixed Banach space $E$, such an extension $\hat{T}$ exists for any choice of $F, G, \phi, T$ if and only if $E=C(X)$ for some Stonian space $X$. They also show that, for a fixed Banach space $F$, such an extension $\hat{T}$ exists for any choice of $E, G, \phi, T$ (that is, $F$ is a $\mathcal{P}_{1}$-space) if and only if $F=C(X)$ for some Stonian space $X$. Furthermore, Kakutani [7] and Bohnenblust [1] prove that, for a fixed Banach space $G$, such an extension exists for any choice of $F, E, \phi, T$ if and only if $G$ is isometric to a Hilbert space.

The corresponding problems for liftings can be formulated as follows: Suppose that $E, F, G$ are Banach spaces, that $\phi$ is a linear mapping of $G$ onto $F$ that maps the closed unit ball in $G$ onto the closed unit ball in $F$ (that is, $\phi$ is a strict metric homomorphism) and that $T: E \rightarrow F$ is a continuous linear mapping. When does $T$ have a norm preserving linear lifting $\hat{T}: E \rightarrow G$, that is, when does there exist a continuous linear mapping $\hat{T}$ : $E \rightarrow G$ such that $\|\hat{T}\|=\|T\|$ and such that the following diagram commutes?

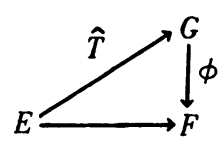

$T$

Figure 2

Grothendieck [5], Pelczynski [11] and Köthe [8] have established the following results concerning this question. For a fixed Banach space $E$, such a lifting $\hat{T}$ exists for arbitrary $F, G, \phi, T$ if and only if $E=l^{1}(\Gamma)$ for some index set $\Gamma$. Moreover, if we add the restrictions that $G, F$ are dual spaces and that $\phi$ is an adjoint mapping, then $l^{1}(\Gamma)$ can be replaced by any $A L$. space. More generally, for a fixed Banach space $E$, a lifting $\hat{T}$ with range in the bidual $G^{\prime \prime}$ exists for arbitrary $F, G, \phi, T$ if and only if $E$ is an $A L$. space (see Figure 3).

In the corresponding extension and lifting problems for Banach lattices $E, F, G$, we consider a positive linear mapping $T$ and we are interested in finding an extension or lifting $\hat{T}$ that is also a positive linear mapping. Moreover, in the extension problem, we require that the isometry $\phi: F \rightarrow G$ is also a lattice homomorphism, while in the lifting problem, we restrict $\phi$ : 


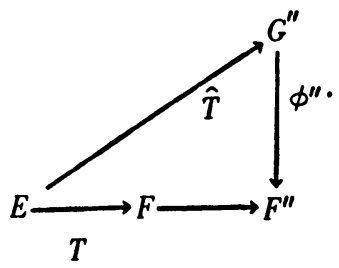

Figure 3

$G \rightarrow F$ to be a metric homomorphism with property $\left(A_{0}\right)$ (see (1.1)). This latter property is dual to the requirement that a mapping be a lattice homomorphism (see (1.2)). We shall call a Banach lattice $E$ injective if for any $F, G$, $\phi, T$ as restricted above, $T$ has a positive linear extension $\hat{T}: G \rightarrow E$ such that $\|\hat{T}\|=\|T\|$. Dually, we say that $E$ is d-projective if for any $F, G, \phi, T$ as restricted above, $T$ has a positive linear lifting $\hat{T}: E \rightarrow G^{\prime \prime}$ such that $\|\hat{T}\|=\|T\|$, that is, there exists $\hat{T}: E \rightarrow G^{\prime \prime}$ such that $\|\hat{T}\|=\|T\|$ and such that the diagram in Figure 3 commutes. (The notion of injective objects is not only a categorical one but depends also on the class of embeddings used (see [15]). The injective objects in the category $\mathrm{Ban}_{1}$ [16] with the isometries as embeddings are the $\mathscr{P}_{1}$-spaces. The injective objects of $\mathrm{Ban}_{\infty}$ with the topological isomorphisms as embeddings are usually called injective Banach spaces. The injective Banach lattices are the injective objects in the category whose objects are the Banach lattices and whose morphisms are the positive contractive linear mappings and where the embeddings are the lattice isometries.)

In $\$ 1$, we establish the duality between lattice homomorphisms and positive linear mappings that preserve order intervals and we also prove an analogue to the Hahn-Banach theorem for positive linear functionals. In $\$ 2$, we show that for a Stonian space $X, C(X)$ is an injective Banach lattice and that an $A L$-space is a $d$-projective Banach lattice. In contrast to the situation in the category of Banach spaces, we prove in $\$ 3$ that any $A M$-space is a $d$-projective Banach lattice and that any $A L$-space is an injective Banach lattice. Then we apply these results in $\$ 4$ to the study of order summable and majorizing linear mappings and the tensor product $E \hat{\otimes}_{|\sigma|} F$ where $E$ is a Banach lattice and $F$ is a Banach space. We show that $E \otimes_{|\sigma|} F$ is injective (that is, $\phi \otimes \psi$ is an isometry whenever $\phi, \psi$ are isometries and $\phi$ is a lattice homomorphism) and projective (that is, $\phi \otimes \psi$ is a metric homomorphism whenever $\phi, \psi$ are metric homomorphisms and $\phi$ has property $\left(A_{0}\right)$ ). Finally, in $\$ 5$, we discuss some results concerning extensions and liftings of positive compact linear mappings.

We conclude this introduction with a summary of the special terminology 
and results concerning Banach lattices and tensor products that will be used throughout this paper. We refer the reader to [12] or [13] for background material on Banach lattices.

Suppose that $E$ is a Banach lattice. Then $E$ is an $A M$-space if $\|x \vee y\|=$ $\sup \{\|x\|,\|y\|\}$ for all $x \geq 0, y \geq 0$ in $E$. An element $e$ of an $A M$-space is a unit if $\|e\|=1$ and $x \leq e$ fce all $x \in E$ such that $\|x\| \leq 1$. $E$ is an $A L$ space if $\|x+y\|=\|x\|+\|y\|$ for all $x \geq 0, y \geq 0$ in $E$. Kakutani has proved that: (a) The norm dual of an $A M$-space is an $A L$-space and that the norm dual of an $A L$-space is an $A M$-space with a unit. (b) If $E$ is an $A M$-space with unit, then $E$ is lattice isometric to the Banach lattice $C(X)$ of continuous real-valued functions on a compact Hausdorff space $X$. (c) If $E$ is an $A L$-space, then $E$ is lattice isometric to the Banach lattice $L^{1}$ of equivalence classes of integrable functions on a suitable measure space. The vector lattice $C(X)$ is order complete if and only if $X$ is Stonian, that is, the closure of every open set in $X$ is open. $X$ is totally disconnected if the topology of $X$ has a base consisting of clopen sets, that is, sets that are both open and closed.

If $E$ is a Banach lattice and $x \geq 0$ in $E$, then $E_{x}$ is the linear hull of the order interval $[-x, x]$ in $E$ equipped with the norm given by the Minkowski functional of $[-x, x] . E_{x}$ is a lattice ideal in $E$ and $E_{x}$ is an AM-space with unit $x$.

If $E$ is a Banach lattice, then a sequence $\left\{x_{n}\right\} \subset E$ is summable if the net $\left\{\Sigma_{n \in H} x_{n}\right\}$ directed by the finite subsets of $H$ of the set $N$ of natural numbers converges in $E$. We write $\sum_{n=1}^{\infty} x_{n}$ for the limit of this net. The space $l^{1}[E]$ of all summable sequences in $E$ equipped with the norm

$$
\left\|\left\{x_{n}\right\}\right\|_{\epsilon}=\sup \left\{\sum_{n=1}^{\infty}\left|\left\langle x_{n}, x^{\prime}\right\rangle\right|: x^{\prime} \in E^{\prime},\left\|x^{\prime}\right\| \leq 1\right\}
$$

is a Banach space; moreover if $x_{n} \geq 0$ for all $n$ then $\left\|\left\{x_{n}\right\}\right\|_{\epsilon}=\left\|\sum_{n=1}^{\infty} x_{n}\right\|$.

Suppose that $E$ is a Banach lattice and that $F$ is a Banach space. A continuous linear mapping $T: E \rightarrow F$ is order summable if there exist an $A L$ space $L$, a positive linear mapping $T_{1}: E \rightarrow L$ and a continuous linear mapping $T_{2}: L \rightarrow F$ such that $T=T_{2} \circ T_{1}$. The infimum of $\left\|T_{1}\right\|\left\|T_{2}\right\|$ taken over all those factorizations of $T$ through $A L$-spaces defines a norm $T \mapsto\|T\|_{L}$ on the vector space $S_{+}(E, F)$ of all order summable linear mappings of $E$ into $F$. If $S: F \rightarrow E$ is a continuous linear mapping, then $S$ is majorizing if there exist an $A M$-space $C$, a positive linear mapping $S_{2}: C \rightarrow$ $E$ and a continuous linear mapping $S_{1}: F \rightarrow C$ such that $S=S_{2} \circ S_{1}$ The infimum of $\left\|S_{1}\right\|\left\|S_{2}\right\|$ taken over all factorizations of $S$ through $A M$-spaces 
defines a norm $S \mapsto\|S\|_{M}$ on the vector space $M_{+}(F, E)$ of all majorizing linear mappings of $F$ into $E . T: E \rightarrow F$ is order summable if and only if its adjoint $T^{\prime}: F^{\prime} \rightarrow E^{\prime}$ is majorizing; moreover, $\|T\|_{L}=\left\|T^{\prime}\right\|_{M}$ in this case. Similarly $S: F \rightarrow E$ is majorizing if and only if $S^{\prime}: E^{\prime} \rightarrow F^{\prime}$ is order summable; in this case we have $\|S\|_{M}=\left\|S^{\prime}\right\|_{L}$. We refer the reader to [G] and [14] for background material on order summable and majorizing linear mappings.

If $E$ is a Banach lattice and if $F$ is a Banach space, we define $|\sigma|$-norm $\|\cdot\|_{|\sigma|}$ on $E \otimes F$ by

$$
\|u\|_{|\sigma|}=\inf \left\{\left\|\sum_{i=1}^{n} x_{i}\right\| y_{i}\|\|: x_{i} \geq 0, u=\sum_{i=1}^{n} x_{i} \otimes y_{i}\right\} .
$$

$E \otimes F$ equipped with the $|\sigma|$-norm will be denoted by $E \otimes|\sigma| F$ and its completion by $E \quad \tilde{\otimes}_{|\sigma|} F$. The dual of $E \tilde{\otimes}_{|\sigma|} F$ is isometric to $S_{+}\left(E, F^{\prime}\right)$ equipped with the norm $\|\cdot\|_{L}$ and also isometric to $M_{+}\left(F, E^{\prime}\right)$ equipped with the norm $\|\cdot\|_{M}$.

If $E$ and $F$ are Banach spaces, then a continuous linear mapping $T$ : $E \rightarrow F$ is a metric homomorphism if it maps the open unit ball in $E$ onto the open unit ball in $F$.

If $E$ and $F$ are Banach lattices and if $T: E \rightarrow F$ is a positive linear mapping then $T$ is strictly positive if $T x=0$ for $x \geq 0$ implies $x=0$.

The author wishes to thank Professor Anthony L. Peressini for all his assistance during the preparation of this paper.

1. In this section, we shall establish two preliminary results that will be needed in our discussion of extensions and liftings of positive linear mappings.

(1.1). Definitions. Suppose that $E, F$ are Banach lattices and that $T$ : $E \rightarrow F$ is a positive linear mapping. Then $T$ has property:

$\left(A_{0}\right)$ if $T[0, x]$ is dense in $[0, T x]$ for each $x \geq 0$ in $E$;

$\left(A_{1}\right)$ if $T$ is a lattice homomorphism;

$\left(\Lambda_{2}\right)$ if $T[0, x]=[0, T x]$ for each $x \geq 0$ in $E$.

The basic relationships between these properties are described in the following result which is essentially due to T. Ando.

(1.2). Proposition. Suppose that $E$ and $F$ are Banach lattices and that $T: E \rightarrow F$ is a positive linear mapping. Then

(a) $T$ has property $\left(A_{0}\right)$ if and only if $T^{\prime}$ has property $\left(A_{1}\right)$;

(b) $T$ has property $\left(A_{1}\right)$ if and only if $T^{\prime}$ has property $\left(A_{2}\right)\left(\operatorname{or}\left(A_{0}\right)\right)$.

Proof. To prove (a), assume that $T$ has property $\left(A_{0}\right)$, that $y^{\prime} \in F^{\prime}$ and that $x \geq 0$ in $E$. Then 


$$
\begin{aligned}
\left(T^{\prime} y^{\prime}\right)^{+}(x) & =\sup _{z \in[0, x]}\left\langle z, T^{\prime} y^{\prime}\right\rangle=\sup _{z \in[0, x]}\left\langle T z, y^{\prime}\right\rangle \\
& =\sup _{y \in[0, T x]}\left\langle y, y^{\prime}\right\rangle \quad\left(\text { by }\left(A_{0}\right)\right) \\
& =\left\langle T x,\left(y^{\prime}\right)^{+}\right\rangle=\left\langle x, T^{\prime} y^{\prime+}\right\rangle .
\end{aligned}
$$

Consequently, $\left(T^{\prime} y^{\prime}\right)^{+}=T^{\prime}\left(y^{\prime+}\right)$ for all $y^{\prime} \in F^{\prime}$, that is, $T^{\prime}$ has property $\left(A_{1}\right)$. Conversely, suppose that $T^{\prime}$ has property $\left(A_{1}\right)$, that $x \geq 0$ in $E$ and that $0 \leq z \notin \overline{T[0, x]}$. Then $z$ and $T[0, x]$ can be strictly separated by a closed hyperplane, that is, there exist $y^{\prime} \in F^{\prime}$ and $\alpha \in \mathbf{R}$ such that $\left\langle w, T^{\prime} y^{\prime}\right\rangle$ $=\left\langle T w, y^{\prime}\right\rangle \leq \alpha\left\langle\left\langle z, y^{\prime}\right\rangle\right.$ for all $w \in[0, x]$. But then $\left\langle T x, y^{\prime+}\right\rangle=\left\langle x, T^{\prime}\left(y^{\prime+}\right)\right\rangle=$ $\left\langle x,\left(T y^{\prime}\right)^{+}\right\rangle \leq \alpha\left\langle\left\langle z, y^{\prime+}\right\rangle\right.$ since $T^{\prime}$ has property $\left(A_{1}\right)$ and since $z \geq 0$. Since $T x \geq 0$ it follows that $z \notin[0, T x]$. Consequently, $T[0, x]$ is dense in $[0, T x]$, that is, $T$ has property $\left(A_{0}\right)$.

If $T$ has property $\left(A_{1}\right)$, and if $y^{\prime} \geq 0$ in $F^{\prime}$, then we shall show that $T^{\prime}\left[0, y^{\prime}\right]=\left[0, T^{\prime} y^{\prime}\right]$. Now $T^{\prime}\left[0, y^{\prime}\right]$ and $\left[0, T^{\prime} y^{\prime}\right]$ are weak*-compact and by an argument similar to that used in the preceding step we can see that $T^{\prime}\left[0, y^{\prime}\right]$ is $\sigma\left(E^{\prime}, E\right)$-dense in $\left[0, T^{\prime} y^{\prime}\right]$. Therefore, $T^{\prime}$ has property $\left(\mathrm{A}_{2}\right)$.

If $T^{\prime}$ has property $\left(A_{0}\right)$, then $T^{\prime \prime}$ has property $\left(A_{1}\right)$ by $(a)$ and so $T$ has property $\left(A_{1}\right)$. This completes the proof.

The following extension theorem follows from (1.1).

(1.3). Corollary. Suppose that $F$ is a closed sublattice of a Banach lattice $E$ and that $y^{\prime}$ is a positive linear functional on $F$. Then $y^{\prime}$ has a positive linear extension $x^{\prime}$ to $E$ such that $\left\|x^{\prime}\right\|=\left\|y^{\prime}\right\|$.

Proof. By the Hahn-Banach theorem, $y^{\prime}$ has a continuous linear extension $z^{\prime}$ to $E$ such that $\left\|z^{\prime}\right\|=\left\|y^{\prime}\right\|$. Since the canonical injection of $F$ into $E$ is a lattice homomorphism, its adjoint, which maps each $w^{\prime} \in E^{\prime}$ onto its restriction $w_{F}^{\prime}$ to $F$, has property $\left(\mathrm{A}_{2}\right)$. Hence, since $\left|z^{\prime}\right|_{F} \geq y^{\prime} \geq 0$, it follows that there is an $x^{\prime} \in\left[0,\left|z^{\prime}\right|\right]$ such that $x_{F}^{\prime}=y^{\prime}$. Since $\left\|y^{\prime}\right\| \leq\left\|x^{\prime}\right\| \leq$ $\left\|\left|z^{\prime}\right|\right\|=\left\|z^{\prime}\right\|=\left\|y^{\prime}\right\|$ the proof is complete.

2. In this section, we shall show that $C(X)$, where $X$ is a Stonian space, is an injective Banach lattice and that any $A L$-space is a $d$-projective Banach lattice.

(2.1). Proposition. Suppose that $X$ is a Stonian space, that $G$ is a Banach lattice, that $F$ is a closed sublattice of $G$, and that $T$ is a positive linear mapping of $F$ into $C(X)$. Then $T$ has a positive linear extension $\hat{T}: G \rightarrow C(X)$ such that $\|\hat{T}\|=\|T\|$. 
Proof. If $l_{X}^{\infty}$ denotes the vector lattice of all bounded families $\left\{z_{t}: t \in X\right\}$ of real numbers indexed by $X$, then the linear mapping $S: C(X) \rightarrow l_{X}^{\infty}$ defined by $S(f)=\{f(t): t \in X\}$ is a (lattice) isometry. Since $C(X)$ is a $\mathscr{P}_{1}$-space there exists a $P: l_{X}^{\infty} \rightarrow C(X)$ with $\|P\|=1$ such that $P \circ S$ is the identity on $C(X)$. $P$ maps the element $\left\{z_{t}\right\}$ with $z_{t}=1$ for all $t \in X$ onto the identically one function $e_{X}$ of $C(X)$. Consequently, $P$ is positive. (In fact, by [3, Theorem 2.5] $P$ can be chosen to be a lattice homomorphism.)

For each $t \in X$, define $x_{t}^{\prime} \in F^{\prime}$ by $x_{t}^{\prime}(x)=(T x)(t)(x \in F)$. Then $x_{t}^{\prime} \geq 0$ and $\left\|x_{t}^{\prime}\right\| \leq\|T\|$ for each $t \in X$. By (1.3), each $x_{t}^{\prime}(t \in X)$ has a positive linear extension $z_{t}^{\prime}$ to $G$ such that $\left\|z_{t}^{\prime}\right\|=\left\|x_{t}^{\prime}\right\|$. Define a positive linear mapping $T_{1}: G \rightarrow l_{X}^{\infty}=C\left(\beta X_{d}\right)$ by $T_{1}(z)=\left\{z_{t}^{\prime}(z): t \in X\right\}$. Clearly, $\left\|T_{1}\right\| \leq$ $\|T\|$ and $T_{1}$ is an extension of $S \circ T$. Hence, the positive linear mapping $\hat{T}: G \rightarrow C(X)$ defined by $\hat{T}=P \circ T_{1}$ provides the required extension of $T$.

It should be remarked that if $X$ is a compact Hausdorff space (or if $E$ is an $A M$-space), then (2.1) implies that each positive linear mapping $T$ of a closed sublattice $F$ of a Banach lattice $G$ into $C(X)$ has a positive linear extension $\hat{T}: G \rightarrow C(X)^{\prime \prime}$ (or $T: G \rightarrow E^{\prime \prime}$ ) such that $\|\hat{T}\|=\|T\|$.

The following result is dual to (2.1).

(2.2). Proposition. Suppose that $L$ is an AL-space, that $G, F$ are Banach lattices, that $\phi: G \rightarrow F$ is a metric homomorphism with property $\left(A_{0}\right)$ and that $T: L \rightarrow F$ is a positive linear mapping. Then there exists a positive linear mapping $\hat{T}: L \rightarrow G^{\prime \prime}$ such that $\|\hat{T}\|=\|T\|$ and such that $\phi^{\prime \prime} \circ \hat{T}=$ $T \circ Q$ where $Q$ is the canonical mapping of $F$ into $F^{\prime \prime}$.

Proof. Since $L$ is an $A L$-space, it follows that $L^{\prime}$ is an order complete $A M$-space and so $L^{\prime}$ is lattice isometric to $C(X)$ for some Stonian space $X$. The adjoint $T^{\prime}$ is a positive linear mapping of $F^{\prime}$ into $L^{\prime}$. Also, since $\phi$ is a metric homomorphism with property $\left(A_{0}\right)$, it follows from (1.2) that $F^{\prime}$ can be identified with a closed sublattice of $G^{\prime}$. By (2.1), $T^{\prime}$ can be extended to a positive linear mapping $S: G^{\prime} \rightarrow L^{\prime}$ such that $\|S\|=\|T\|$. The restriction of $S^{\prime}: L^{\prime \prime} \rightarrow G^{\prime \prime}$ to $L$ then provides a positive linear extension $\hat{T}: L \rightarrow$ $G^{\prime \prime}$ of $T$ such that $\|\hat{T}\|=\|T\|$ and $\phi^{\prime \prime} \circ \hat{T}=T \circ Q$.

We remark that if we assume, in addition to the hypotheses of (2.2), that $G, F$ are dual Banach lattices and that $\phi$ is an adjoint mapping, then there is a positive linear lifting $\hat{T}: L \rightarrow G$ of $T$ such that $\|\hat{T}\|=\|T\|$.

3. The main objective of this section is to show that any AM-space is a d-projective Banach lattice and that any $A L$-space is an injective Banach lattice. The following preliminary result is a special type of lifting theorem for the identity mapping on $C(Y)$ for a totally disconnected compact Hausdorff space 
$Y$. In its proof we shall make use of the fact that if $x$ is a positive element of a Banach lattice $E$, then $y$ is an extreme point of $[0, x]$ if and only if $y \wedge(x-y)=0$. The set of extreme points of a set $A$ will be denoted by Ext $A$.

(3.1). Proposition. Suppose that $X, Y$ are compact Hausdorff spaces and that $Y$ is totally disconnected. If $T: C(X) \rightarrow C(Y)$ is a strictly positive linear mapping with property $\left(\mathrm{A}_{2}\right)$ and if $T e_{X}=e_{Y}$ where $e_{X}, e_{Y}$ are the identically one functions on $X, Y$, then there is a lattice isometry $S: C(Y)$ $\rightarrow C(X)$ such that $S e_{Y}=e_{X}$ and $T \circ S=\mathrm{id}_{C(Y)^{\circ}}$

Proof. We begin by showing that each extreme point of the order interval $\left[0, e_{Y}\right]$ is the image of a unique extreme point of $\left[0, e_{X}\right]$. Suppose $f \epsilon$ Ext $\left[0, e_{Y}\right]$. Since $T$ has property $\left(A_{2}\right)$ and since $T e_{X}=e_{Y}$, it follows that there is a $g \in\left[0, e_{X}\right]$ such that $T g=f$. If $0 \leq b \leq g \wedge\left(e_{X}-g\right)$, then $0 \leq$ $T h \leq f \wedge\left(e_{Y}-f\right)=0$; consequently, $g \wedge\left(e_{X}-g\right)=0$ since $g$ is strictly positive. Therefore, $g \in \operatorname{Ext}\left[0, e_{X}\right]$. If $g_{1}$ is another element of $\operatorname{Ext}\left[0, e_{X}\right]$ such that $T g_{1}=f$, then since $g \vee g_{1}+g \wedge g_{1}=g+g_{1}$, it follows that $f=$ $1 / 2\left\{T\left(g \vee g_{1}\right)+T\left(g \wedge g_{1}\right)\right\}$. But $f \in \operatorname{Ext}\left[0, e_{Y}\right]$ and $T\left(g \vee g_{1}\right), T\left(g \wedge g_{1}\right)$ belong to $\left[0, e_{Y}\right]$ so $f=T\left(g \vee g_{1}\right)=T\left(g \wedge g_{1}\right)$, that is, $T\left(g \vee g_{1}-g \wedge g_{1}\right)=$ 0. Since $T$ is strictly positive, it follows that $g \vee g_{1}=g \wedge g_{1}$ so $g=g_{1}$.

Since $f \in \operatorname{Ext}\left[0, e_{Y}\right]$ if and only if $f \wedge\left(e_{Y}-f\right)=0$, it follows that Ext $\left[0, e_{X}\right]$ coincides with the set of characteristic functions of all clopen sets in $Y$. Hence, $\operatorname{Ext}\left[0, e_{Y}\right]$ is a Boolean algebra with respect to the lattice operations of $C(Y) . T$ is a one-to-one mapping of $B=\left\{g \in \operatorname{Ext}\left[0, e_{X}\right]\right.$ : $\left.T_{g} \in \operatorname{Ext}\left[0, e_{Y}\right]\right\}$ onto $A=\operatorname{Ext}\left[0, e_{Y}\right]$. Let $S_{0}: A \rightarrow B$ be the inverse of $T$.

We shall now show that $B$ is a Boolean algebra for the lattice operations induced by $C(X)$ and that $S_{0}$ is a Boolean isomorphism. Clearly, $S_{0}(0)=0$ and $S_{0} e_{Y}=e_{X}$. If $f_{1}, f_{2}$ are disjoint elements of $A$, then $S_{0} f_{1} \wedge S_{0} f_{2}=0$ since $T$ is strictly positive. But then

$$
T\left(S_{0} f_{1} \vee S_{0} f_{2}\right)=T\left(S_{0} f_{1}+S_{0} f_{2}\right)=f_{1}+f_{2}=f_{1} \vee f_{2}
$$

so that $S_{0}\left(f_{1} \vee f_{2}\right)=S_{0} f_{1} \vee S_{0} f_{2}$ for all $f_{1}, f_{2}$ in $A$. Consequently, $S_{0}$ is a Boolean isomorphism of the Boolean algebra $A$ onto $B$.

The linear hull $M$ of $A$ is a sublattice of $C(Y)$ and $S_{0}$ extends to a linear lattice isometry $S$ of $M$ into $C(X)$. Moreover, since $Y$ is totally disconnected, $M$ is dense in $C(Y)$. Consequently, $S$ extends to a lattice isometry of $C(Y)$ into $C(X)$ and, since $(T \circ S)(f)=f$ for each $f \in \operatorname{Ext}\left[0, e_{Y}\right]$, it follows that $T \circ S=\mathrm{id}_{C(Y)^{\circ}}$ 
Corollary. Suppose that $X$ is a Stonian space, that $Y$ is a compact Hausdorff totally disconnected space and that $T: C(X) \rightarrow C(Y)$ is a positive order continuous linear mapping with property $\left(\mathrm{A}_{2}\right)$ such that $T e_{X}=e_{Y}$. Then there exists a lattice isometry $S: C(Y) \rightarrow C(X)$ such that $T \circ S=\mathrm{id}_{C(Y)^{\circ}}$

Proof. If $I=\{f \in C(X): T|f|=0\}$, then $I$ is a band in $C(X)$ since $T$ is positive and order continuous. Therefore, there is a clopen subset $X_{0}$ of $X$ such that $f \in I$ if and only if $f$ vanishes off $X_{0}$. If $X_{1}$ is the complement of $X_{0}$ in $X$ and if $T_{1}$ is the mapping induced on $C\left(X_{1}\right)$ by $T$, then $T_{1}$ satisfies the hypotheses of (3.1), so there is a lattice isometry $S_{1}$ of $C(Y)$ into $C\left(X_{1}\right)$ such that $S_{1} e_{Y}=e_{X_{1}}$ and $T_{1} \circ S_{1}=\mathrm{id} C(Y)^{\circ}$. But then if $S_{1}$ is composed with the canonical mapping of $C\left(X_{1}\right)$ into $C(X)$, we obtain a lattice isometry $S: C(Y) \rightarrow C(X)$ of the required sort.

We can now show that $A L$-spaces are injective Banach lattices.

(3.2). Proposition. Suppose that $L$ is an AL-space, that $F$ is aclosed sublattice of a Banach lattice $G$ and that $T: F \rightarrow L$ is a positive linear mapping. Then $T$ has a positive linear extension $\hat{T}: G \rightarrow L$ such that $\|\hat{T}\|=$ $\|T\|$.

Proof. If $\phi: F \rightarrow G$ is the canonical injection, then $\phi^{\prime}$ and $T^{\prime}$ are positive linear mappings and $\phi^{\prime}$ is order continuous. Since $L$ is an $A L$. space, there is a Stonian space $X$ such that $L^{\prime}$ is lattice isometric to $C(X)$. If $T^{\prime} e_{X}=x^{\prime}$, then $x^{\prime} \geq 0$ and $x^{\prime}$ has a positive linear extension $y^{\prime}$ to $G$ such that $\left\|y^{\prime}\right\|=\left\|x^{\prime}\right\|$ by (1.3). Then $F_{x}^{\prime}$, and $G_{y}^{\prime}$, are order complete $A M$. spaces, so there exist Stonian spaces $Y$ and $Z$ such that $F_{x}^{\prime}$, is lattice isometric to $C(Y)$ and $G_{y^{\prime}}^{\prime}$ is lattice isometric to $C(Z)$. The restriction $R$ of $\phi^{\prime}$ to $C(Z)$ is an order continuous, positive linear mapping of $C(Z)$ onto $C(Y)$ such that $\operatorname{Re}_{Z}=e_{Y}$. Moreover, since $\phi$ is a lattice homomorphism and since $F_{x}^{\prime}, G_{y}^{\prime}$, are lattice ideals in $F^{\prime}, G^{\prime}$, respectively, then $R$ has property $\left(A_{2}\right)$ by (1.3). Therefore, by (3.1) Corollary, there exists a lattice isometry $S: C(Y) \rightarrow C(Z)$ such that $R \circ S=\mathrm{id}_{C(Y)^{\circ}}$. The mapping $S \circ T^{\prime}$ is a positive linear mapping of $C(X)=L^{\prime}$ into $G^{\prime}$ such that $\left\|S \circ T^{\prime}\right\|=\left\|y^{\prime}\right\|=\|T\|$. Since it is well known that there is a positive projection $P$ of norm 1 from $L^{\prime \prime}$ onto $L$, we can take $\hat{T}$ to be $P \circ\left[\left.\left(S \circ T^{\prime}\right)^{\prime}\right|_{F}\right]$ to obtain the desired extension.

The preceding construction of $\hat{T}$ is illustrated by the following commutative diagram.

Corollary 1. If $F$ is a closed sublattice of a Banach lattice $G$ and if $F$ is an AL-space, then $F$ is the range of a positive projection on $E$ of norm 1. 


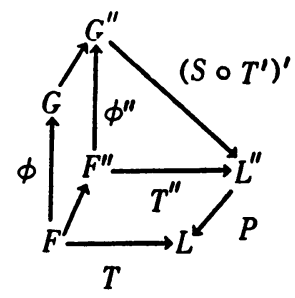

Figure 4

Proof. The identity mapping $\mathrm{id}_{F}$ on $F$ is positive and has norm 1 so (3.2) implies that $\mathrm{id}_{F}$ extends to a positive linear mapping $P: G \rightarrow F$ of norm 1. Clearly, $P$ is a projection with range $F$.

Corollary 2 (Dean [2]). Any closed sublattice of an AL-space is the range of a positive projection of norm 1 .

Proof. Corollary 2 is a special case of Corollary 1.

Corollary 3. If $E$ and $G$ are Banach lattices, if $F$ is a closed sublattice of $G$ that is an AL-space, and if $T: F \rightarrow E$ is a positive linear mapping, then $T$ has a positive linear extension $\hat{T}: G \rightarrow E$ such that $\|\hat{T}\|=\|T\|$.

Proof. This follows immediately from the fact that $F$ is the range of a positive projection on $G$ of norm 1 .

The following result, which is dual to (3.2), shows that any $A M$-space is a $d$-projective Banach lattice.

(3.3). Proposition. Suppose that $M$ is an AM-space, that $\phi$ is a metric homomorphism with property $\left(A_{0}\right)$ from a Banach lattice $G$ onto a Banach lattice $F$ and that $T: M \rightarrow F$ is a positive linear mapping. Then there exists a positive linear mapping $\hat{T}: M \rightarrow G^{n}$ such that $\phi^{\prime \prime} \circ \hat{T}=Q \circ T$ and $\|\hat{T}\|=\|T\|$, where $Q$ is the canonical mapping of $F$ into $F^{\prime \prime}$.

Corollary 1. If $X$ is a compact Hausdorff space and if $\phi$ is a metric homomorphism with property $\left(A_{0}\right)$ from a Banach lattice $G$ onto $C(X)$, then $C(X)^{\prime \prime}$ is lattice isometric to an order complete sublattice of $G^{\prime \prime}$ which is the range of a positive projection of norm 1.

Corollary 2. If $X$ is a compact Hausdorff space and if $\phi$ is a metric homomorphism with property $\left(\mathrm{A}_{0}\right)$ from $C(X)$ onto a Banach lattice $E$, then $E^{\prime \prime}$ is lattice isometric to an order complete sublattice of $C(X)^{n}$ which is the range of a positive projection of norm 1.

Note that under the conditions of Corollary 2, $E$ is lattice isometric to $C(Y)$ for some compact Hausdorff space $Y$. 
Corollary 3. If $E$ and $G$ are Banach lattices, if $X$ is a compact Hausdorff space and if $\phi$ is a metric homomorphism with property $\left(A_{0}\right)$ from $G$ onto $C(X)$, then every positive linear mapping $T: E \rightarrow C(X)$ has a positive linear lifting $\hat{T}: E \rightarrow G^{\prime \prime}$ such that $\|\hat{T}\|=\|T\|$.

If we strengthen the hypotheses of (3.2) and Corollary 3 by requiring the domain and range of $\phi$ to be dual Banach lattices and $\phi$ to be an adjoint mapping, then we can conclude that the lifting $\hat{T}$ of $T$ has its range in $G$ rather than $G^{\prime \prime}$.

It is easy to see that if $F$ is a closed sublattice of an injective Banach lattice $E$ that is the range of a positive contractive projection then $F$ is injective. Also, if $\left\{E_{\alpha}\right\}$ is a family of injective Banach lattices then their ${ }^{\infty}$-product $\left\{\left(x_{a}\right): x_{a} \in E_{a}\right.$, sup $\left.\left\|x_{a}\right\|<\infty\right\}$ equipped with the product order and the norm $\left\|\left(x_{a}\right)\right\|=\sup \left\{\left\|x_{a}\right\|\right\}$ is an injective Banach lattice.

(3.4). Lemma. Any Banach lattice $E$ is lattice isometric to a closed sublattice of an $T^{\circ}$-product of AL-spaces (and hence of an injective Banach lattice).

Proof. If $0 \leq x^{\prime} \in E^{\prime}$, then $I=\left\{x:\left\langle|x|, x^{\prime}\right\rangle=0\right\}$ is an ideal in $E$. The functional $(x+I) \mapsto\left\langle|x|, x^{\prime}\right\rangle$ defines a lattice norm on $E / I$ that is additive on the positive cone, so its completion is an $A L$-space which we denote by $\left(E, x^{\prime}\right)$. The canonical lattice homomorphism $\phi_{x^{\prime}}$ from $E$ into $\left(E, x^{\prime}\right)$ has norm $\left\|x^{\prime}\right\|$. Obviously, the map $x \rightarrow\left(\phi_{x}(x)\right)$ defines a lattice isometry fromı $E$ into the $l^{\infty}$-product of the family $\left\{\left(E, x^{\prime}\right): 0 \leq x^{\prime} \in E,\left\|x^{\prime}\right\|=1\right\}$.

(3.5). Proposition. Let $E$ be a Banach lattice. Then the following assertions are equivalent:

(a) $E$ is an injective Banach lattice.

(b) If $E$ is lattice isometrically embedded in a Banach lattice $F$ then there exists a positive contractive projection from $F$ onto $E$.

(c) $E$ is lattice isometric to a closed sublattice of an $l^{\infty}$-product of $A L$-spaces that is the range of a positive contractive projection.

Proof. The implication (a) $\Rightarrow(b)$ is trivial. The implication (b) $\Rightarrow$ (c) follows from (3.4) and the implication (b) $\Rightarrow(a)$ follows from (3.2) and the remarks above.

4. As applications of the preceding results, we shall now establish an extension theorem for order summable linear mappings, a dual lifting theorem for majorizing linear mappings, and the injectivity and projectivity of the $|\sigma|$ norm on the tensor product of a Banach lattice with a Banach space. 
(4.1). Proposition. Suppose that $G$ is a Banach lattice, that $E$ is a closed sublattice of $G$, and that $F$ is a Banach space. If $T: E \rightarrow F$ is an order summable linear mapping, then $T$ can be extended to an order summable linear mapping $\hat{T}: G \rightarrow F$ such that $\|\hat{T}\|_{L}=\|T\|_{L}$.

Proof. If $T$ is order summable then there exist an $A L$-space $L$, a positive linear mapping $T_{1}: E \rightarrow L$ with $\left\|T_{1}\right\|=\|T\|_{L}$ and a linear mapping $T_{2}$ : $L \rightarrow G$ with $\left\|T_{2}\right\| \leq 1$ (see $[14,1.6]$ ). By (3.2), $T_{1}$ has a positive linear extension $\hat{T}_{1}: G \rightarrow L$ such that $\left\|\hat{T}_{1}\right\|=\left\|T_{1}\right\|$. But then $\hat{T}=T_{2} \circ \hat{T}_{1}$ is a positive linear extension of $T$ which is order summable and $\|\hat{T}\|_{L} \leq\left\|\hat{T}_{1}\right\|=$ $\|T\|_{L}$. Since $\hat{T}$ is an extension of $T$ we have $\|\hat{T}\|_{L} \geq\|T\|_{L}$ which completes the proof.

The following lifting theorem for majorizing mappings is dual to (4.1).

(4.2). Proposition. Suppose that $F$ and $G$ are Banach lattices, that $\phi$ is a metric homomorphism of $G$ onto $F$ with property $\left(A_{0}\right)$. If $T: E \rightarrow F$ is a majorizing linear mapping, then there exists a majorizing linear mapping $\hat{T}: E \rightarrow G^{n}$ with $\|\hat{T}\|_{M}=\|T\|_{M}$ and such that $\phi^{\prime \prime} \circ \hat{T}=Q \circ T$ where $Q$ is the canonical mapping of $F$ into $F^{\prime \prime}$.

Proof. $T^{\prime}: F^{\prime} \rightarrow E^{\prime}$ is an order summable linear mapping since $T$ is majorizing. Also, since $\phi$ is a positive metric homomorphism of $G$ onto $F$, $\phi^{\prime}$ is a lattice isometry of $F^{\prime}$ into $G^{\prime}$ by (1.2). Hence, by (4.1), there exists an order summable linear mapping $\hat{S}: G^{\prime} \rightarrow E^{\prime}$ such that $\hat{S} \circ \phi^{\prime}=T^{\prime}$ and such that $\|\hat{S}\|_{L}=\left\|T^{\prime}\right\|_{L}=\|T\|_{M}$. But then the restriction $\hat{T}$ of $\hat{S}^{\prime}$ to $E$ is a majorizing linear mapping of $E$ into $G^{\prime \prime}$ such that $\|\hat{T}\|_{M}=\|T\|_{M}$ and $Q \circ T=$ $\phi^{\prime \prime} \circ \hat{T}$ where $Q$ is the canonical mapping of $F$ into $F^{\prime \prime}$.

As with (2.2) and Corollary 3 of (3.2), we can conclude that the lifting $\hat{T}$ of $T$ described in (4.2) is a majorizing linear mapping of $E$ into $G$ provided that we assume, in addition to the hypotheses of (4.2), that $G, F$ are dual Banach lattices and $\phi$ is an adjoint mapping.

We now establish the injectivity and projectivity of the $|\sigma|$-norm on the tensor product of a Banach lattice with a Banach space.

(4.3). Proposition. Suppose that $E, G$ are Banach lattices, that $F, H$ are Banach spaces, that $\phi: E \rightarrow G$ is a lattice isometry and that $\psi: F \rightarrow H$ is an isometry. Then $\phi \otimes \psi: E \widetilde{\otimes}_{|\sigma|} F \rightarrow G \widetilde{\otimes}_{|\sigma|} H$ is an isometry.

Proof. It suffices to prove that the adjoint mapping $(\phi \otimes \psi)^{\prime}$ is a metric homomorphism of $\left(G \widetilde{\otimes}_{|\sigma|} H\right)^{\prime}=\left(S_{+}\left(G, H^{\prime}\right),\|\cdot\|_{L}\right)$ onto $\left(E \otimes_{|\sigma|} F\right)^{\prime}=$ $\left(S_{+}\left(E, F^{\prime}\right),\|\cdot\|_{L}\right)$. Hence, suppose that $U \in S_{+}\left(E, F^{\prime}\right)$ and that $\|U\|_{L}<1$. 
Then $U=U_{2} \circ U_{1}$ where $U_{1}$ is a positive linear mapping of $E$ into an $A L$ space $L$ and $U_{2}$ is a continuous linear mapping of $L$ into $F^{\prime}$ such that $\left\|U_{1}\right\|\left\|U_{2}\right\|<1$. Since $\phi$ is a lattice isometry, $\phi(E)$ is a closed sublattice of $G$. Hence, by (3.2) there exists a positive linear mapping $V_{1}: G \rightarrow L$ such that $\left\|V_{1}\right\|=\left\|U_{1}\right\|$ and $V_{1} \circ \phi=U_{1}$. Also, $\psi^{\prime}$ is a metric homomorphism of $H^{\prime}$ onto $F^{\prime}$ so there is a continuous linear mapping $V_{2}: L \rightarrow H^{\prime}$ such that $\psi^{\prime} \circ V_{2}=U_{2}$ and $\left\|V_{2}\right\|=\left\|U_{2}\right\|$ (see Grothendieck [s]). But then $V=$ $V_{2} \circ V_{1} \in S_{+}\left(G, H^{\prime}\right),(\phi \otimes \psi)^{\prime} V=U$ and $\|V\|_{L} \leq\left\|V_{2}\right\|\left\|V_{1}\right\|<1$, so $(\phi \otimes \psi)^{\prime}$ is a metric homomorphism. This completes the proof.

(4.4). Proposition. Suppose that $E_{0}$ is a closed subspace of a Banach lattice $E$ for which $G=E / E_{0}$ is a Banach lattice for the canonical quotient ordering and that the quotient mapping $\phi: E \rightarrow G$ has property $\left(A_{0}\right)$, that $F_{0}$ is a closed subspace of a Banach space $F$ and that $\psi: F \rightarrow H=F / F_{0}$ is the canonical quotient mapping. Then $\phi \otimes \psi$ is a metric homomorphism of $E \widetilde{\otimes}_{|\sigma|} F$ onto $G \widetilde{\otimes}_{\mid \text {| }} H$.

Proof. It suffices to show that $(\phi \otimes \psi)^{\prime}$ is an isometry of $\left(M_{+}\left(H, G^{\prime}\right)\right.$, $\left.\|\cdot\|_{M}\right)$ into $\left(M_{+}\left(F, E^{\prime}\right),\|\cdot\|_{M}\right)$. Hence, suppose that $T \in M_{+}\left(H, G^{\prime}\right),\|T\|_{M}=$ 1. Then $\phi^{\prime}: G^{\prime} \rightarrow E^{\prime}$ is an order continuous lattice isomorphism and $(\phi \otimes \psi)^{\prime}(T)=\phi^{\prime} \circ T \circ \psi$ is a majorizing mapping of $F$ into $E^{\prime}$ by Corollary 5 of (1.7) in [14]. Now $\psi$ maps the open unit ball $U$ in $F$ onto the open unit ball $V$ in $H$, so the supremum of the set $T(V)$ in $G^{\prime}$ coincides with the supremum of the set $\left(\phi^{\prime} T \psi\right)(U)$ in $E^{\prime}$ since $\phi^{\prime}$ is an order continuous lattice isomorphism. Therefore

$$
\left\|(\phi \otimes \psi)^{\prime}(T)\right\|_{M}=\left\|\sup \left(\phi^{\prime} T \psi\right)(U)\right\|_{E^{\prime}}=\|\sup T(V)\|_{G^{\prime}}=\|T\|_{M}
$$

since $\phi^{\prime}$ is an isometry.

5. In this section, we consider the problem of obtaining positive compact linear extensions and liftings of positive compact linear mappings. The following result is basic to these considerations.

(5.1). Proposition. Suppose that $E$ and $F$ are Banach lattices and that $\phi$ is a metric homomorphism from $E$ onto $F$ with property $\left(A_{2}\right)$. If $\left\{y_{n}\right\}$ is a positive summable sequence in $F$ such that $\left\|\left\{y_{n}\right\}\right\|_{\epsilon}<1$, then there exists a positive summable sequence $\left\{x_{n}\right\}$ in $E$ such that $\left\|\left\{x_{n}\right\}\right\|_{\epsilon}<1$ and $\phi\left(x_{n}\right)=y_{n}$ for all $n$.

Proof. The proof relies on the following lemma.

Lemma. If $E, F$ and $\phi$ are restricted as in (5.1) and if $0 \leq y_{i} \in F$ for $i=1$, 
$2, \ldots, n$ with $\left\|\sum_{i=1}^{n} y_{i}\right\|<1$, then there exist $0 \leq x_{i} \in E$ for $i=1,2, \ldots, n$ such that $\left\|\Sigma_{i=1}^{n} x_{i}\right\|<1$ and $\phi\left(x_{i}\right)=y_{i}$ for $i=1,2, \ldots, n$.

To verify the lemma, we first note that if $y=\sum_{i=1}^{n} y_{i}$, then there is an $x_{0} \in E$ such that $\left\|x_{0}\right\|<1$ and $\phi\left(x_{0}\right)=y$ since $\phi$ is a metric homomorphism. But then $\phi\left(\left|x_{0}\right|\right) \geq y \geq 0$ so there is an $x \in\left[0,\left|x_{0}\right|\right]$. such that $\phi(x)=y$ since $\phi$ has property $\left(\mathrm{A}_{2}\right)$; clearly; $\|x\|<1$. Now suppose that $1 \leq m<n$ and that there exist $0 \leq x_{j} \in E$ for $j<m$ such that $\Sigma_{j<m} x_{j} \leq x$ and $\phi\left(x_{j}\right)=y_{j}$ for $j<m$. Then $\phi$ maps $\left[0, x-\Sigma_{j<m} x_{j}\right]$ onto $\left[0, y-\Sigma_{j<m} y_{j}\right]$ since $\phi$ has property $\left(A_{2}\right)$. Thus, since $y_{m} \leq y-\Sigma_{j<m} y_{j}$, there is an $x_{m}$ in $[0, x-$ $\left.\Sigma_{j<m} x_{j}\right]$ such that $\phi\left(x_{m}\right)=y_{m}$. Clearly $\Sigma_{j \leq m} x_{j} \leq x$ so that $\left\|\Sigma_{j \leq m} x_{j}\right\|<1$. Hence, by induction the lemma follows.

To complete the proof of the proposition, choose $\delta>0$ so that $\left\|\left\{y_{n}\right\}\right\|_{\epsilon}<$ $1-\delta$. Choose an increasing sequence $\left\{x_{k}\right\}$ of positive integers such that

$$
\left\|\sum_{i=1}^{n_{1}} y_{i}\right\|<1-\delta,\left\|\sum_{n_{k}<i \leq n_{k+1}} y_{i}\right\|<\frac{\delta}{2^{k}}
$$

for $k=1,2, \ldots$. By the above lemma, there exists a positive sequence $\left\{x_{n}\right\}$ in $E$ such that $\phi\left(x_{n}\right)=y_{n}$ for all $n$ and such that

$$
\left\|\sum_{i=1}^{n_{1}} x_{i}\right\|<1-\delta,\left\|\sum_{n_{k}<i \leq n_{k}+1} x_{i}\right\|<\frac{\delta}{2^{k}}
$$

for $k=1,2, \ldots$. Since the norm of $E$ is monotone on the positive cone, $\left\{x_{n}\right\}$ is a summable sequence in $E$ with $\left\|\left\{x_{n}\right\}\right\|_{\epsilon}<1$. This completes the proof of the proposition.

(5.2). Proposition. Suppose that $E$ and $F$ are Banach lattices and that $\phi$ is a metric homomorphism of $F$ onto $E$ with property $\left(\mathrm{A}_{2}\right)$. If $T: c_{0} \rightarrow E$ is a positive compact linear mapping such that $\|T\|<1$, then there is a positive compact linear mapping $\hat{T}: c_{0} \rightarrow F$ such that $\|\hat{T}\|<1$ and $T=\phi \circ \hat{T}$.

Proof. Let $e_{n}$ denote the $n$th unit vector in $c_{0}$ and let $x_{n}=T e_{n}$ for each positive integer $n_{0}$. Then $x_{n} \geq 0$ for all $n$ since $T$ is positive. Moreover, $\left\{x_{n}\right\}$ is summable in $E$ since $T$ is compact and $\left\|\left\{x_{n}\right\}\right\|_{\epsilon}<1$ since $\|T\|<1$. By (5.1), there exists a positive summable sequence $\left\{y_{n}\right\}$ in $F$ such that $\left\|\left\{y_{n}\right\}\right\|_{\epsilon}<1$ and such that $\phi\left(y_{n}\right)=x_{n}$ for all $n_{0}$. Define $\hat{T}: c_{0} \rightarrow F$ by

$$
\hat{T}\left(\lambda_{n}\right)=\sum_{n=1}^{\infty} \lambda_{n} y_{n} \quad\left(\lambda_{n}\right) \in c_{0} .
$$

Then it is easy to verify that $\hat{T}$ is a positive compact linear mapping with $\|\hat{T}\|<1$ and that $\phi \circ \hat{T}=T$. 
By duality, we obtain the following extension theorem from (5.2).

(5.3). Proposition. Suppose that E, F are Banach lattices and that $\phi: E \rightarrow F$ is a lattice isometry. If $T: E \rightarrow l^{1}$ is a positive compact linear mapping such that $\|T\|<1$, then $T$ has a positive compact linear extension $\hat{T}: F \rightarrow l^{1}$ such that $\|\hat{T}\|<1$.

(5.4). Proposition. Suppose that $E$ is a Banach lattice and that $F$ is a closed sublattice of $E$. If $T$ is a positive compact linear mapping from $E$ into $c_{0}$ then $T$ has a positive compact linear extension $\hat{T}$ with $\|\hat{T}\|=\|T\|$.

Proof. Let $y_{n}^{\prime}=T^{\prime} e_{n}$ where $e_{n}$ denotes the $n$th unit vector in $l^{1}$. Since $T$ is compact $\lim \left\|y_{n}^{\prime}\right\|=0$ and since $T$ is positive $y_{n}^{\prime} \geq 0$. By (1.2) there exists a sequence $\left\{x_{n}^{\prime}\right\} \subset E^{\prime}$ of positive elements such that $x_{n}^{\prime}$ is an extension of $y_{n}^{\prime}$ with $\left\|x_{n}^{\prime}\right\|=\left\|y_{n}^{\prime}\right\|$. Since $\lim \left\|x_{n}^{\prime}\right\|=0$ the linear mapping $\hat{T}: x \rightarrow\left(\left\langle x, x_{n}^{\prime}\right\rangle\right)$ is a positive compact extension of $T$. Moreover, $\|\hat{T}\|=$ $\sup \left\|x_{n}^{\prime}\right\|=\sup \left\|y_{n}^{\prime}\right\|=\|T\|$.

We conclude this section with some open questions concerning positive compact mappings:

(1) In (5.2) and (5.3), can $c_{0}$ be replaced by an arbitrary $A M$-space and $l^{1}$ by an arbitrary $A L$-space?

An affirmative answer to (1) would be implied by an affirmative answer to the following question:

(2) If $T$ is a positive compact linear mapping of a Banach lattice $E$ into an $A L$-space $L$ then does $T$ have a representation $T x=\sum_{n=1}^{\infty}\left\langle x, x_{n}^{\prime}\right\rangle y_{n^{\prime}}$ $x \in E$, where $\left\|y_{n}\right\|=1$ for all $n, y_{n} \geq 0$, and $\left\{x_{n}^{\prime}\right\}$ is a positive summable sequence in $E^{\prime}$ ? (An affirmative answer to this question would imply that every positive compact operator $T: C(X) \rightarrow L$ is nuclear.)

(3) If $E$ is a closed sublattice of a Banach lattice $F$, if $X$ is a compact Hausdorff space and if $T: E \rightarrow C(X)$ is a positive compact linear mapping, then can $T$ be extended to a positive compact linear mapping $\hat{T}$ : $F \rightarrow C(X)$ ?

\section{REFERENCES}

1. H. F. Bohnenblust, $A$ characterization of complex Hilbert spaces, Portugal. Math. 3 (1942), 103-109. MR 4, 247.

2. D. W. Dean, Direct factors of $(A L)$-spaces, Bull. Amer. Math. Soc. 71 (1965), 369-371. MR $31 \# 5057$.

3. A. M. Gleason, Projective topological spaces, Illinois J. Math. 2 (1958), 482-489. MR 22 \#12509.

4. D. B. Goodner, Projections in normed linear spaces, Trans, Amer, Math, Soc. 69 (1950), 89-108. MR 12, 266.

5. A. Grothendieck, Une caractérisation vectorielle-métrique des espaces $L^{1}$, Canad. J. Math. 7 (1955), 552-561. MR 17, 877. 
6. H. Jacobs, Ordered topological tensor products, Disser tation, University of Illinois, 1969.

7. S. Kakutani, Some characterizations of Euclidean space, Japan. J. Math. 16 (1939), 93-97. MR 1, 146.

8. G. Köthe, Hebbare lokalkonvexe Räume, Math. Ann, 165 (1966), 188-195. MR 33 \#4651.

9. J. Lo Kelley, Banach spaces with the extension property, Trans, Amer, Math. Soc, 72 (1952), 323-326. MR 13, 659.

10. L. Nachbin, A theorem of Hahn-Banach type for linear transformations, Trans, Amer, Math. Soc, 68 (1950), 28-46. MR 11, 369.

11. A. Pelczyński, Projections in certain Banach spaces, Studia Math, 19 (1960), 209-228. MR 23 \#A3441.

12. A. L. Peressini, Ordered topological vector spaces, Harper \& Row, New York, 1967. MR 37 \#3315.

13. H. H. Schaefer, Topological vector spaces, Macmillan, New York, 1966. MR 33 \#1689.

14. U. Schlotterbeck, Über Klassen majorisierbarer Operatoren auf Banachverbänden, Revo Acad. Ci. Zaragoza 26 (1971), 585-614.

15. Z. Semadeni, Projectivity, injectivity and duality, Rozprawy Mat. 35 (1963), 47 pp. MR 27 \#4776.

16. Banach spaces of continuous functions. Vol. I, Monografie Mato, Tom 55, PWN, Warsaw, 1971. MR 45 \#5730.

DEPARTMENT OF MATHEMATICS, UNIVERSITY OF ILLINOIS, URBANA, ILLINOIS 61801 